Radial and Nonradial Pulsations as Probes of Stellar Physics

ASP Conference Series, Vol. 259, 2002

C. Aerts, T.R. Bedding, \& J. Christensen-Dalsgaard, eds.

\title{
Asteroseismology of $\beta$ Cep: Influence of Updated Microphysics
}

\author{
H. Shibahashi, T. Koshikawa ${ }^{1}$ \\ Department of Astronomy, University of Tokyo, Japan
}

\begin{abstract}
With updated microphysics such as opacity, we revise the result of asteroseismic investigations for $\beta$ Cephei and show the tolerable range of the physical parameters of the star.
\end{abstract}

$\beta$ Cep is a pulsating star showing variations in luminosity and velocity. Telting et al. (1997) deduced from the line profile variability that the variations show periodicity with four frequencies, being part of a quintuplet with equal spacing. Three frequencies among them are separated from the main frequency, $f_{1}$, which has been identified as the radial mode. In addition to these, an independent frequency, $f_{2}$, was found and it has been identified as a quadrupole mode.

Besides the pulsations, a variable magnetic field with a period of 12 days has been reported by Henrichs et al. (2000). The UV wind lines of this star also reveal a periodic variation with both a 6 day and a 12 day periodic component (Henrichs et al., 1993). From these results, it is concluded that the magnetic field of $\beta$ Cep is oblique to the rotation axis.

Shibahashi \& Aerts (2000) interpreted the observed equidistant fine structure of the frequency spectrum as a manifestation of a magnetic perturbation of an eigenmode, which would be a radial mode in the absence of the magnetic field. With the interpretation of an independent quadrupole mode, $f_{2}$, they deduced the mass, evolutionary stage, rotational frequency, magnetic field strength, and geometrical configuration of $\beta$ Cep. In this paper, we update the microphysics and the evolutionary code, and re-deduce the physical quantities of this star by a seismic approach.

By sweeping out evolutionary models of various masses, we search for stellar models whose radial and quadrupole mode eigenfrequencies coincide with the observed frequencies $f_{1}$ and $f_{2}$. The left panel of Fig. 1 shows the series of these models on the H-R diagram, calculated by using the updated evolutionary program $\left(X_{0}=0.70\right.$ and $\left.Z_{0}=0.03\right)$. The microphysics adopted in this updated code is as follows; opacity: OPAL, nuclear reaction: Bahcall, equation of state: OPAL. The solid lines show the models whose radial mode frequency coincides with $f_{1}$, and the dashed lines show the models in which the quadrupole mode has the same frequency as $f_{2}$. $\beta$ Cep must be on one of the crossing points of the solid and dashed lines. The candidate is (A) an $\sim 8 M_{\odot}$ star at the late stage of the hydrogen core-burning phase, $(\mathrm{B})$ a $\sim 12 M_{\odot}$ star near the turning point, or (C) $\mathrm{a} \sim 18 M_{\odot}$ star at the middle of the hydrogen core-burning phase.

\footnotetext{
${ }^{1}$ Present address: Department of Earth and Planetary Science, University of Tokyo, Japan
} 

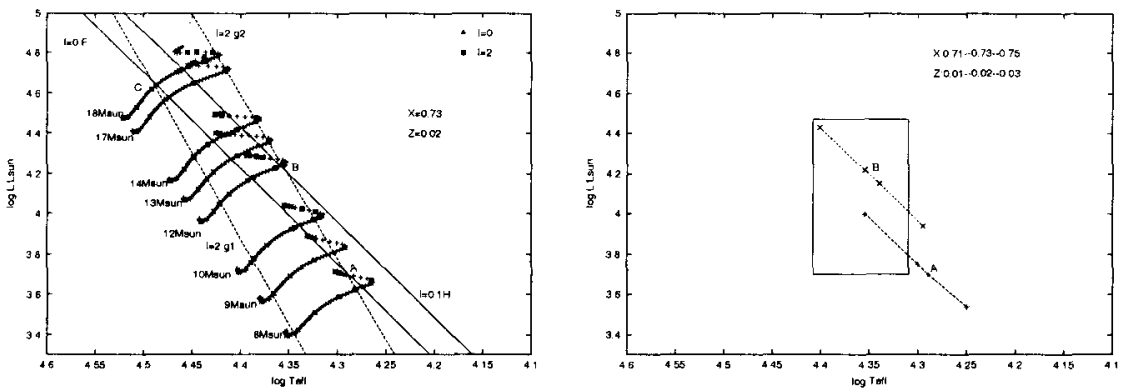

Figure 1. Left: HR diagram of 8-18 $M_{\odot}$ stars. The stars whose radial mode frequency coincides with $f_{1}$ are connected with the solid lines, and those whose quadrupole mode frequency matches with $f_{2}$ are shown by the dashes lines. The crossing points, $\mathrm{A}-\mathrm{C}$, are the candidate locations for $\beta$ Cep. Right: The loci of the crossing points, $A$ and $\mathrm{B}$, with the change of $X_{0}$ and $Z_{0}$. The tick marks on each sequence indicate models with $\left(X_{0}, Z_{0}\right)=(0.71,0.01),(0.73,0.02),(0.75,0.02)$, and $(0.75,0.03)$, from the left to the right. The box indicates the possible range of $\beta$ Cep estimated with the HIPPARCOS data.

We change the values of the parameters $X_{0}$ and $Z_{0}$ to see their effects on the asteroseismically deduced parameters. We repeat a series of the stellar evolution calculation and the frequency calculation for all the combinations of $X_{0}=0.71,0.73,0.75$ and $Z_{0}=0.01,0.02$, and 0.03 . The crossing points on the H-R diagram shown in the left panel of Fig. 1 shift systematically with the change in the parameter $\left(X_{0}, Z_{0}\right)$. Since the parameter range is narrow enough, each of the crossing points is expected to form a rectangle with the change in the parameter space. Actually the expected rectangle on the H-R diagram crashes and it almost looks like a line segment as shown in the right panel of Fig. 1.

Combination of the distance data measured by HIPPARCOS with the apparent magnitude of the star and the spectral data leads independently to a plausible range of $\beta$ Cep on the H-R diagram. The box shown in the right panel of Figure 1 is the range determined in this way. This constraint rules out the candidate $\mathrm{C}$ and only the candidates $\mathrm{A}$ and $\mathrm{B}$ remain; that is, $\beta$ Cep must be an $\sim 8 M_{\odot}$ star at the late stage of the hydrogen core-burning phase or a $\sim 12 M_{\odot}$ star near the turning point.

\section{References}

Henrichs, H.F., et al. 1993, in New perspectives on stellar pulsation and pulsating variable stars, eds. J. Nemec \& J. Matthews, (Cambridge Univ. Press), 295

Henrichs, H.F., et al. 2000, in Magnetic fields of chemically peculiar and related stars, eds. Y. Glagolevskij \& I. Romanyuk, 57

Shibahashi, H. \& Aerts, C. 2000, ApJ, 531, L143

Telting, J.H., Aerts, C., \& Mathias, P. 1997, A\&A, 322, 493 